\title{
Diagnóstico da doença de Chagas: Avaliação da reação cruzada em pacientes com leishmaniose visceral
}

\author{
Serological diagnosis of Chagas disease: Evaluation of cross reaction in patients with visceral \\ leishmaniasis
}

Diagnóstico serológico de la enfermedad de Chagas: Evaluación de la reacción cruzada en pacientes con leishmaniasis visceral

Lucas Silva Barcelos

ORCID: https://orcid.org/0000-0001-8835-533X Universidade do Oeste Paulista, Brasil

E-mail: lucassilva_barcelos@hotmail.com Amanda Aparecida Silva de Aguiar ORCID: https://orcid.org/0000-0003-3856-0620 Universidade do Oeste Paulista, Brasil E-mail: amanda_daguiar@hotmail.com Fernando Nunes Gavioli Boni ORCID: https://orcid.org/0000-0002-6594-1157 Universidade do Oeste Paulista, Brasil E-mail: boni_fernando@outlook.com

Luiz Euribel Prestes Carneiro

ORCID: https://orcid.org/0000-0002-4577-1525 Universidade do Oeste Paulista, Brasil E-mail: luizepcarneiro@gmail.com

Thaís Batista de Carvalho

ORCID: https://orcid.org/0000-0003-0306-3077 Universidade do Oeste Paulista, Brasil E-mail: thais@unoeste.br

Eliana Peresi-Lordelo

ORCID: https://orcid.org/0000-0002-5320-6825 Universidade do Oeste Paulista, Brasil E-mail: lordeloeliana@gmail.com

\begin{abstract}
Resumo
No Brasil, existem diversas regiões endêmicas simultaneamente para leishmaniose e doença de Chagas e como os agentes causadores pertencem ambos à mesma família, Trypanosomatidae, eles compartilham diversos antígenos, que no momento do diagnóstico sorológico, podem promover reações cruzadas. Diversos autores avaliaram a reação cruzada Chagas/leishmaniose em cães, entretanto poucos trabalhos fizeram este questionamento em humanos. O objetivo do presente estudo foi identificar a possibilidade de reação cruzada para o diagnóstico da doença de Chagas em pacientes com leishmaniose visceral curada. Para tanto, foram analisadas 30 amostras de soro de pacientes com leishmaniose visceral curada atendidos no Ambulatório de Infectologia do Hospital Regional de Presidente Prudente, São Paulo. As amostras foram avaliadas em duplicata através de três kits sorológicos para o diagnóstico da doença de Chagas: ensaio imunoenzimático (ELISA), imunofluorescência indireta (IFI) e hemaglutinação indireta (HAI). Das 30 amostras analisadas, apenas o soro de um paciente mostrou-se reagente na IFI, entretanto, a duplicata avaliada não foi positiva, assim como os resultados de ELISA e HAI. No presente estudo, devido à reatividade para a IFI apresentar apenas um T. cruzi marcado, com a sua duplicata e outros testes sorológicos (ELISA e HAI) negativos, podemos concluir que o paciente com leishmaniose visceral apresentou uma reação cruzada para o diagnóstico da Doença de Chagas.
\end{abstract}

Palavras-chave: Trypanosoma cruzi; Leishmania; Testes sorológicos; Reações cruzadas.

\section{Abstract}

In Brazil, there are several simultaneously endemic regions for leishmaniasis and Chagas disease and, as the causative agents both belong to the same family, Trypanosomatidae, they share several antigens, which at the time of serological diagnosis can promote cross reactions. Several authors have evaluated Chagas/leishmaniasis cross reaction in dogs, however few studies have made this question in humans. The objective of this study was to identify the possibility of cross reaction for the diagnosis of Chagas disease in patients with cured visceral leishmaniasis. 
Therefore, thirty serum samples from patients with cured visceral leishmaniasis at the Infectious Diseases Clinic of the Hospital Regional de Presidente Prudente, São Paulo were analyzed. The samples were evaluated using three serological kits for the diagnosis of Chagas disease: immunoenzymatic assay (ELISA), indirect immunofluorescence (IFI) and indirect hemagglutination (HAI). Of the 30 samples analyzed, only one patient's serum showed to be reagent in the IFI, however, the duplicate evaluated was not positive, as well as the ELISA and HAI results. In the present study, due to the reactivity for the IFI to present in only one marked $T$. cruzi, with its duplicate and other serological tests (ELISA and HAI) negative, we can conclude that the patient with visceral leishmaniasis presented a cross reaction for the diagnosis of Chagas disease.

Keywords: Trypanosoma cruzi; Leishmania; Serologic tests; Cross reactions.

\section{Resumen}

En Brasil, existen varias regiones endémicas simultáneamente para la leishmaniasis y la enfermedad de Chagas y, como los agentes causales pertenecen a la misma familia, Trypanosomatidae, comparten varios antígenos, que en el momento del diagnóstico serológico pueden promover reacciones cruzadas. Varios autores han evaluado la reacción cruzada de Chagas / leishmaniasis en perros, sin embargo pocos estudios han hecho esta pregunta en humanos. El objetivo de este estudio fue identificar la posibilidad de reacción cruzada para el diagnóstico de la enfermedad de Chagas en pacientes con leishmaniasis visceral curada. Por lo tanto, se analizaron treinta muestras de suero de pacientes con leishmaniasis visceral curada en la Clínica de Enfermedades Infecciosas del Hospital Regional de Presidente Prudente, São Paulo. Las muestras se evaluaron mediante tres kits serológicos para el diagnóstico de la enfermedad de Chagas: ensayo inmunoenzimático (ELISA), inmunofluorescencia indirecta (IFI) y hemaglutinación indirecta (HAI). De las 30 muestras analizadas, solo el suero de un paciente mostró ser reactivo en el IFI, sin embargo, el duplicado evaluado no fue positivo, así como los resultados de ELISA y HAI. En el presente estudio, debido a la reactividad para que el IFI se presente en un solo T. cruzi marcado, con su duplicado y otras pruebas serológicas (ELISA y HAI) negativas, podemos concluir que el paciente con leishmaniasis visceral presentó una reacción cruzada para el diagnóstico de la enfermedad de Chagas.

Palabras clave: Trypanosoma cruzi; Leishmania; Pruebas serológicas; Reacciones cruzadas.

\section{Introdução}

De acordo com a Organização Mundial de Saúde, cerca de 6 a 7 milhões de pessoas estão infectadas por Trypanosoma cruzi, distribuindo-se, principalmente, em áreas endêmicas de 21 países da América Latina, incluindo o Brasil, onde a transmissão vetorial ainda apresenta relevância (WHO, 2018).

Assim como a doença de Chagas, a leishmaniose também é uma doença zoonótica de extrema importância no Brasil, podendo se apresentar sob três formas: visceral, cutânea e mucocutânea. A leishmaniose visceral ou calazar é endêmica em 62 países, sendo que 200 milhões de pessoas apresentam risco de contrair a doença. Leishmania infantum chagasi é o protozoário causador do calazar no novo mundo, particularmente na América do Sul (Ferreira-Silva et al., 2017).

Tanto a doença de Chagas quanto à leishmaniose visceral são parasitoses que apresentam um caráter negligenciado, afetando geralmente as populações mais carentes e marginalizadas (WHO, 2013; Paho, 2020). No Brasil, existem diversas regiões endêmicas simultaneamente para ambas as parasitoses e como os agentes causadores apresentam proximidade filogenética, acabam compartilhando diversos antígenos. Dessa forma, no momento do diagnóstico sorológico, considerado padrão ouro nas análises clínicas, podem ocorrer reações cruzadas, especialmente quando se utilizam pools de complexos antigênicos (Gontijo \& Melo, 2004).

Na doença de Chagas, a via de transmissão natural ocorre pelo inseto da subfamília Triatominae que ao realizar a hematofagia, lesiona a pele, permitindo a entrada da forma tripomastigota metacíclica eliminada em suas fezes. Atualmente, está em evidência a transmissão por via oral associada a surtos, onde são consumidos alimentos contaminados com os dejetos do inseto ou até mesmo o inseto infectado. Até o momento, os alimentos mais associados a esse tipo de transmissão foram o açaí e o caldo de cana. Como o T. cruzi é um parasita sanguíneo, a doença de Chagas também pode ser transmitida de forma congênita ou por doação de sangue, para tanto, os serviços de pré-natal e doação de sangue realizam exames de diagnóstico com princípio imunológico como triagem (Dias et al., 2016). 
A via de transmissão da leishmaniose ocorre através da picada de fêmeas de flebotomíneos infectados ${ }^{7}$ para o ser humano e outras espécies de mamíferos, principalmente o cão. No Brasil, estudo associou a transfusão sanguínea como um fator de risco para a presença de anticorpos anti-leishmania em pacientes politransfundidos e em hemodiálise (Luz et al., 1997). Outros autores demonstraram a presença do DNA do parasita em doadores de sangue assinomáticos, entretanto, não é realizado nenhum tipo de triagem nos hemocentros para essa parasitose (Silva et al., 2020; França et al., 2018).

Devido ao fato dos agentes causadores da doença de Chagas e leishmaniose pertencerem à mesma família, eles partilham inúmeros antígenos que podem promover reações cruzadas durante o diagnóstico sorológico (Caballero et al, 2007). Diversos autores avaliaram a reação cruzada Chagas/leishmaniose em cães, entretanto poucos estudos fizeram este questionamento em humanos (Mascolli et al, 2016; Luciano et al., 2009; Perez et al., 2016; Umezawa et al., 1999). Desse modo, o presente trabalho teve como objetivo identificar uma possível reação cruzada para o diagnóstico da doença de Chagas em pacientes com leishmaniose visceral curada.

\section{Metodologia}

Este é um estudo primário e transversal, no qual foram analisadas 30 amostras de soro de pacientes com leishmaniose visceral curada atendidos no Ambulatório de Infectologia do Hospital Regional de Presidente Prudente/SP. Os pacientes foram diagnosticados por quadro clínico-epidemiológico e/ou diagnóstico imunológico por ensaio imunoenzimático (ELISA) ou imunofluorescência indireta (IFI) para leishmaniose. Todos os pacientes receberam tratamento e foram considerados curados para a leishmaniose visceral. No momento da coleta de sangue para as análises, a média de tempo de tratamento ficou em torno de 59,48 meses, sendo o período mínimo de 12 meses e máximo de 108 meses. O presente projeto recebeu aprovação do comitê de ética, sob protocolo CAAE:13352319.0.0000.5515.

Foram utilizados três kits sorológicos para o diagnóstico da doença de Chagas: ELISA recombinante v 3.0 (Wiener), hemaglutinação indireta (HAI - WAMA) e IFI (Imuno-Con Chagas - WAMA). Todos os testes foram lidos e interpretados no dia da sua realização, conforme as normas do fabricante, para não haver interferências nos resultados. Os testes foram realizados em duplicata para a IFI e em triplicata para o ELISA e a HAI.

\section{Resultados}

Dentre as amostras avaliadas dos pacientes com leishmaniose visceral curada, apenas uma evidenciou reatividade para a IFI, apresentando apenas um T. cruzi totalmente marcado, incluindo o seu contorno, por uma intensa fluorescência verdeamarelada (Figura 1), entretanto, o resultado não pode ser observado na duplicata. Além disso, os demais testes sorológicos (HAI e ELISA) para o mesmo paciente positivo na IFI foram não reagentes. Estes resultados sugerem que houve uma reação cruzada para a Doença de Chagas na reatividade da IFI apresentada. As amostras de soro dos outros pacientes avaliados no estudo apresentaram-se negativas para T. cruzi em todos os testes sorológicos utilizados. 
Figura 1. Teste de imunofluorescência indireta reagente para Trypanosoma cruzi de paciente com leishmaniose visceral curada observado em microscópio óptico de fluorescência em aumento de 400x.

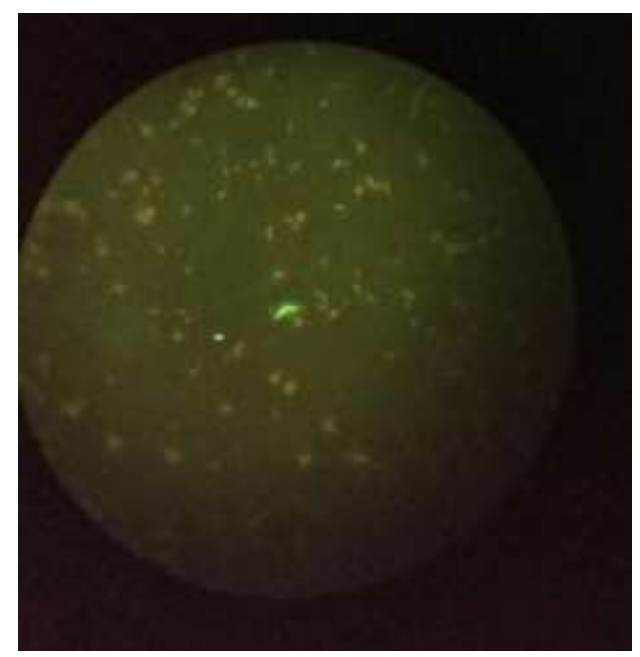

Fonte: Autores (2021).

\section{Discussão}

Diversos estudos verificaram um aumento da sensibilidade do ELISA para T. cruzi utilizando-se um homogenato de antígenos do parasita, entretanto, também se verificou a falta de especificidade em amostras de pacientes com leishmaniose (Dias at al., 2016; Toledo et al., 2017; Caballero et al., 2007; Mascolli et al, 2016). Como não existe um padrão ouro para discriminar a infecção pelo T. cruzi da Leishmania, a especificidade dos kits é avaliada utilizando-se soros de pacientes com leishmaniose de áreas consideradas "Chagas-free" (Umezawa et al., 2004; Aguire et al., 2006; Vega Benedetti et al., 2013). Entretanto, esta metodologia apresenta viés, pois as variáveis que influenciam a reação cruzada de uma região, como prevalência dos tripanossomatídeos, infecções concomitantes e hábitos nutricionais, geralmente são diferentes de outra região a ser avaliada (Matos et al., 2015).

Assim, a interpretação de resultados de testes diagnósticos em áreas endêmicas para a doença de Chagas e leishmaniose deve ser cautelosa. Estudo realizado no estado de Minas Gerais que avaliou pacientes com doença de Chagas observou reatividade do ELISA para leishmaniose visceral em quatro casos, sendo que, três destes foram confirmados para leishmaniose visceral por western blotting (WB), revelando a coexistência de ambos tripanossomatídeos no mesmo paciente (Ferreira-Silva et al., 2017).

Um relato de caso realizado por Matos e colaboradores (2015) que ocorreu no município de Bacarena/PA, obteve os resultados de HAI, IFI IgM e IFI IgG todos reagentes para Chagas e elevados títulos de IFI IgG para leishmaniose. Baseado na elevada titulação para Leishmania e nos sinais clínicos do paciente, este estudo permitiu concluir que houve reação cruzada para Chagas. Entretanto, o estudo não utilizou técnicas confirmatórias, como WB ou PCR, para descartar uma possível coinfecção entre Chagas/leishmaniose.

No Brasil, existem diversas regiões com elevada prevalência de leishmaniose e Chagas, como os estados de Piauí, Tocantins, Pará, Mato Grosso do Sul, Minas Gerais, São Paulo e outras áreas do sul do país (MS, 2014; Martins-Melo et al., 2014). A cidade de Presidente Prudente, interior do estado de São Paulo, faz fronteira com estados das regiões centro oeste e sul com prevalência de ambas as doenças, além de estar localizada na rota para a dispersão da leishmaniose visceral (Oliveira et al., 2016). Essa cidade também é considerada um ambiente urbano com transmissão recente e alguns casos reportados de leishmaniose visceral humana, associados principalmente com locais de elevada taxa de leishmaniose visceral canina (PrestesCarneiro et al., 2019). 
Estudo realizado no Hemocentro de Ribeirão Preto no período de 2012 a 2014 demonstrou que de um total de 281.551 doações, 64.654 foram de doadores de sangue pela primeira vez, apresentando uma soroprevalência para Chagas de 0,15\%, com confirmação pelo teste de IFI de 0,10\% dos casos. No município de Presidente Prudente, cidade filial do Hemocentro de Ribeirão Preto, foram observados 10.289 doadores de sangue primário, com $0,11 \%$ de soroprevalência para Chagas e $0,04 \%$ dos casos confirmados por IFI. Desta forma, os autores concluíram que a prevalência de anticorpos anti-T. cruzi, no período de 2012 a 2014, foi de 0,10\%, na parte ocidental do Estado de São Paulo (Slavov et al., 2017). Portanto, mesmo que a região estudada seja endêmica para a leishmaniose visceral, não se pode descartar a possibilidade uma coinfecção Chagas/leishmaniose ou de uma reação cruzada entre ambas as parasitoses.

\section{Considerações Finais}

No presente estudo, devido à reatividade para a IFI apresentar apenas um T. cruzi marcado, com a sua duplicata e outros testes sorológicos (ELISA e HAI) negativos, podemos concluir que o paciente com leishmaniose visceral apresentou uma reação cruzada para o diagnóstico da Doença de Chagas.

Fica evidente que em regiões endêmicas para a leishmaniose visceral e a Doença de Chagas é importante investigar os pacientes para ambas as doenças, através dos testes sorológicos e técnicas confirmatórias, como o WB e/ou PCR. Além disso, seria recomendável, para melhor avaliar este tipo de população, a realização de uma hemocultura em meio LIT (Liver Infusion Triptose), meio de cultura que mimetiza o intestino do triatomíneo e possibilita a replicação do parasita, favorecendo a pesquisa de tripanossomatídeos, e, posterior avaliação por PCR, para a identificação do gênero do parasita.

\section{Agradecimentos}

À Associação Prudentina de Educação e Cultura (Apec/Unoeste) pelo suporte financeiro.

\section{Referências}

Aguirre, S., Silber, A. M., Brito, M. E., Ribone, M. E., Lagier, C. M., \& Marcipar, I. S. (2006). Design, construction, and evaluation of a specific chimeric antigen to diagnose chagasic infection. Journal of clinical microbiology, 44(10), 3768-3774. https://doi.org/10.1128/JCM.01043-06

Caballero, Z. C., Sousa, O. E., Marques, W. P., Saez-Alquezar, A., \& Umezawa, E. S. (2007). Evaluation of serological tests to identify Trypanosoma cruzi infection in humans and determine cross-reactivity with Trypanosoma rangeli and Leishmania spp. Clinical and vaccine immunology: CVI, 14(8), $1045-1049$. https://doi.org/ 10.1128/CVI.00127-07

Dias, J. C. P. et al. (2016). Consenso Brasileiro em Doença de Chagas. Epidemiol Serv Saúde, 25(esp), 7-86. https://doi.org/10.5123/S167949742016000500002 .

Ferreira-Silva, M. M., Carvalho, S. F. G., Lula, J. F., Teles, L. de F., Basques, F. V., Teixeira, L. A. S., Tibúrcio, Monique Gomes Sales \& Moraes-Souza, H. (2017). Evaluation of cross reactivity between Trypanosoma cruzi and Leishmania infantum in serologically ineligible blood donors due to chagas disease. Rev Patol Trop., 46(1), 113-119. https://doi.org/10.5216/rpt.v46i1.46298

França, A. O., Pompilio, M. A., Pontes, E., de Oliveira, M. P., Pereira, L., Lima, R. B., Goto, H., Sanchez, M., Fujimori, M., Lima-Júnior, M., Matos, M., \& Dorval, M. (2018). Leishmania infection in blood donors: A new challenge in leishmaniasis transmission? PloS one, 13(6), e0198199.

https://doi.org/10.1371/journal.pone.0198199

Gontijo, C. M. F. \& Melo, M. N. (2004). Visceral Leishmaniasis in Brazil: current status, challenges and prospects. Rev Bras Epidemiol, 7(3), 338-49.

Luciano, R. M., Lucheis, S. B., Troncarelli, M. Z., Luciano, D. M., \& Langoni, H. (2009). Cross reaction evaluation of Leishmania spp and Trypanosoma cruzi antigens in dogs' serologic response by indirect immunofluorescence test (IIF). Brazilian Journal of Veterinary Research and Animal Science, 46(3), 181-187. https://doi.org/10.1590/S1415-790X2004000300011

Luz, K. G., da Silva, V. O., Gomes, E. M., Machado, F. C., Araujo, M. A., Fonseca, H. E., Freire, T. C., d'Almeida, J. B., Palatnik, M., \& Palatnik-de Sousa, C. B. (1997). Prevalence of anti-Leishmania donovani antibody among Brazilian blood donors and multiply transfused hemodialysis patients. The American journal of tropical medicine and hygiene, 57(2), 168-171. https://doi.org/10.4269/ajtmh.1997.57.168

Martins-Melo, F. R., Ramos, A. N., Jr, Alencar, C. H., \& Heukelbach, J. (2014). Prevalence of Chagas disease in Brazil: a systematic review and metaanalysis. Acta tropica, 130, 167-174. https://doi.org/10.1016/j.actatropica.2013.10.002

Mascolli, R., Soto, F. R. M., Pinheiro, S. R., Ito, F. H., Gil, A., Azevedo, S. S., Lima, V. M. F., Langoni, H., Fernandes, A. R. F. \& Vasconcellos, S. A. (2016). 
Prevalence and risk factors for leishmaniasis and Chagas disease in the canine population of the tourist city of Ibiúna, São Paulo, Brazil. Semina: Ciencias Agrarias, 37(4), 1971-80. http://dx.doi.org/10.5433/1679-0359.2016v37n4p1971

Matos, H. J., Pinto, A. Y. N., Miranda, A. M. M., Silva, F. L. C. \& Ramos, F. L. P. (2015). Reação cruzada nos testes sorológ icos entre doença de Chagas e leishmaniose visceral em regiões endêmicas para ambas as doenças. Revista Pan-Amazônica de Saúde, 6(1), 65-68. http://scielo.iec.gov.br/scielo.php?script=sci_arttext\&pid=S2176-62232015000100007\&lng=pt\&tlng=pt

Ministério da Saúde (MS). Manual de vigilância e controle da Leishmaniose Visceral. http://www.saude.ba.gov.br/wp-content/uploads/2019/05/2014-Manualde-vigil\%C3\%A2ncia-e-controle-da-leishmaniose-visceral.pdf

Oliveira, A. M., Vieira, C. P., Dibo, M. R., Guirado, M. M., Rodas, L., \& Chiaravalloti-Neto, F. (2016). Dispersal of Lutzomyia longipalpis and expansion of canine and human visceral leishmaniasis in São Paulo State, Brazil. Acta tropica, 164, 233-242. http://dx.doi.org/10.1016/j.actatropica.2016.09.014

Pan American Health Organization (PAHO). (2020). 10 vector-borne diseases that put the population of the Americas at risk. https://www.paho.org/hq/index.php?option=com_content\&view=article\&id=9438:2014-10-vector-borne-diseases-that-put-population-americas-atrisk\&Itemid=135\&lang $=$ pt

Perez, T. D., Figueiredo, F. B., Velho Junior, A. A. M., Silva, V. L., Madeira, M. F., Brazil, R. P. \& Coura, J. R. (2016). Prevalence of american trypanosomiasis and leishmaniases in domestic dogs in a rural area of the municipality of São João do Piauí, Piauí State, Brazil. Revista do Instituto de Medicina Tropical de São Paulo, 58(79), Epub November 03. https://doi.org/10.1590/S1678-9946201658079

Prestes-Carneiro, L. E., Daniel, L., Almeida, L. C., D'Andrea, L. Z., Vieira, A. G., Anjolete, I. R., André, L., \& Flores, E. F. (2019). Spatiotemporal analysis and environmental risk factors of visceral leishmaniasis in an urban setting in São Paulo State, Brazil. Parasites \& vectors, 12(1), 251. https://doi.org/10.1186/s13071-019-3496-6

Silva, L. P., Montenegro, S., Werkauser, R., Sales, K. G. S., Soares, F. C. S., Costa, V. M. A., Bezerra, A. C., Pinto, M. B. A., Ferreira, S. M., Neitzke-Abreu, H. C., Dantas-Torres, F. \& Lima Junior, M. S. C. (2020). Asymptomatic Leishmania infection in blood donors from a major blood bank in Northeastern Brazil: a cross-sectional study. Revista do Instituto de Medicina Tropical de São Paulo, 62, e92. https://doi.org/10.1590/s1678-9946202062092

Slavov, S. N., Otaguiri, K. K., Pinto, M. T., Valente, V. B., Ubiali, E. M., Covas, D. T., \& Kashima, S. (2017). Prevalence of Trypanosoma Cruzi antibodies in blood donors from the Sao Paulo State, Brazil, between 2012 and 2014. Journal of infection in developing countries, 11(3), 277-281. https://doi.org/10.3855/jidc. 8169

Toledo, C. R. S., Almeida, A. S., Chaves, S. A. M., Sabroza, P. C., Toledo, L. M. \& Caldas, J. P. (2017). Vulnerability to the transmission of human visceral leishmaniasis in a Brazilian urban area. Revista de Saúde Pública, 51, 49. http://dx.doi.org/10.1590/s1518-8787.2017051006532

Umezawa, E. S., Bastos, S. F., Camargo, M. E., Yamauchi, L. M., Santos, M. R., Gonzalez, A., Zingales, B., Levin, M. J., Sousa, O., Rangel-Aldao, R., \& da Silveira, J. F. (1999). Evaluation of recombinant antigens for serodiagnosis of Chagas' disease in South and Central America. Journal of clinical microbiology, 37(5), 1554-1560. https://doi.org/10.1128/JCM.37.5.1554-1560.1999

Umezawa, E. S., Luquetti, A. O., Levitus, G., Ponce, C., Ponce, E., Henriquez, D., Revollo, S., Espinoza, B., Sousa, O., Khan, B., \& da Silveira, J. F. (2004). Serodiagnosis of chronic and acute Chagas' disease with Trypanosoma cruzi recombinant proteins: results of a collaborative study in six Latin American countries. Journal of clinical microbiology, 42(1), 449-452. https://doi.org/10.1128/jcm.42.1.449-452.2004

Vega Benedetti, A. F., Cimino, R. O., Cajal, P. S., Juarez, M., Villalpando, C. A., Gil, J. F., Marcipar, I. S., Krolewiecki, A. J., \& Nasser, J. R. (2013). Performance of different Trypanosoma cruzi antigens in the diagnosis of Chagas disease in patients with American cutaneous leishmaniasis from a co-endemic region in Argentina. Tropical medicine \& international health: TM \& IH, 18(9), 1103-1109. https://doi.org/10.1111/tmi.12144

World Health Organization (WHO). (2013). Department of Control of Neglected Tropical Diseases. Sustaining the drive to overcome the global impact of neglected tropical diseases: second WHO report on neglected tropical diseases. file://C:/Users/TEMP.LAPTOPUCL41193/Downloads/9789241564540 eng.pdf

World Health Organization (WHO). Chagas disease (American trypanosomiasis). 2020. https://www.who.int/health-topics/chagas-disease\#tab=tab_1 